\section{$\underset{\substack{\text { hommes } \\ \text { \& migrations }}}{ }$}

\section{Hommes \& migrations}

Revue française de référence sur les dynamiques

migratoires

$1284 \mid 2010$

Migrations et environnement

\title{
Soigner les étrangers en situation irrégulière
}

Des politiques migratoires aux postures professionnelles

\section{Myriam Hachimi Alaoui et Alexandra Nacu}

\section{Q OpenEdition \\ 1 Journals}

\section{Édition électronique}

URL : http://journals.openedition.org/hommesmigrations/1263

DOI : 10.4000/hommesmigrations. 1263

ISSN : 2262-3353

\section{Éditeur}

Musée national de l'histoire de l'immigration

\section{Édition imprimée}

Date de publication : 1 mars 2010

Pagination : 163-173

ISSN : 1142-852X

\section{Référence électronique}

Myriam Hachimi Alaoui et Alexandra Nacu, « Soigner les étrangers en situation irrégulière », Hommes \& migrations [En ligne], 1284 | 2010, mis en ligne le 24 mars 2014, consulté le 22 septembre 2020. URL: http://journals.openedition.org/hommesmigrations/1263; DOI : https://doi.org/10.4000/

hommesmigrations.1263

Ce document a été généré automatiquement le 22 septembre 2020.

Tous droits réservés 


\title{
Soigner les étrangers en situation irrégulière
}

Des politiques migratoires aux postures professionnelles

\author{
Myriam Hachimi Alaoui et Alexandra Nacu
}

1 En Europe, dans un contexte de durcissement généralisé des contrôles en matière d'immigration clandestine, les étrangers en situation irrégulière peinent à accéder aux soins. Pris entre l'urgence humanitaire que suscite leur condition et l'arsenal juridique déployé par les États vis-à-vis des sans-papiers, l'exercice de leur droit à la santé dépend en grande partie de la rigueur et du dévouement des personnels de soins. En France, en Grande-Bretagne et en Suisse, enquête sur les dilemmes du corps médical dans son face-à-face avec les exigences du droit.

2 Le droit à la santé est sans doute celui dont le non-respect suscite les réactions les plus vives. Les États européens se sont engagés à le défendre dans une série de déclarations et de traités internationaux qui font référence aux droits de l'Homme. Au plan interne, la Commission nationale consultative des droits de l'Homme (CNCDH), dans son avis sur la préservation de la santé de 2006, réaffirme que la jouissance des droits fondamentaux doit être déconnectée des préoccupations des politiques migratoires. Pourtant, de nombreuses enquêtes françaises et européennes sur la question de l'accès aux soins des sans-papiers témoignent des difficultés persistantes à faire valoir ce droit. En réalité, l'universalité des droits de l'homme, proclamée sur le plan national et international se voit contrecarrée par les politiques de "maîtrise des flux migratoires". En outre, la portée pratique des textes internationaux et nationaux connaît des limites supplémentaires lorsqu'il s'agit plus spécifiquement d'étrangers en situation irrégulière ${ }^{1}$. Leurs droits butent inévitablement sur l'illégalité du séjour. Les pays de l'Union européenne sont ainsi confrontés à des contradictions notables entre des impératifs humanitaires et les logiques de fermeture et de contrôle de l'espace Schengen. Plusieurs auteurs ont souligné le caractère d'exceptionnalité dont jouit la santé vis-à-vis des biens humains qu'une société démocratique se doit de protéger. Reste que les politiques nationales de santé tendent de manière générale à définir des catégories toujours plus resserrées de bénéficiaires, laissant tous les autres à la marge. 
3 La France, la Grande-Bretagne et la Suisse ont tissé des rapports à l'Autre fondés surdes philosophies de la citoyenneté spécifiques à chacun. Cependant, par-delà ces différences principielles, les politiques d'immigration convergent actuellement dans un même sens : un durcissement des conditions d'entrée sur le territoire, des exigences accrues en matière d'intégration, et une crispation, voire une "obsession" ${ }^{2}$ concernant l'immigration irrégulière. C'est dans cette configuration que se pose la question de l'accès aux soins des étrangers en situation irrégulière et qu'il convient de s'interroger sur la manière dont elle affecte le quotidien du personnel soignant ${ }^{3}$.

\section{Vers une restriction de l'accès aux soins des étrangers en situation irrégulière}

4 Dans les trois pays comparés, on trouve des dispositifs réservés à l'accès aux soins des étrangers en situation irrégulière qui se caractérisent à la fois par de grandes dissemblances et par une convergence autour d'une même tendance à restreindre de facto cet accès.

5 En France, les étrangers en situation irrégulière peuvent bénéficier de l'aide médicale d'État (AME) instituée le $1^{\text {er }}$ janvier 2000 lors de l'entrée en vigueur de la loi du 27 juillet 1999 qui met en place la couverture maladie universelle (CMU), laquelle permet à toute personne résidant en France de façon stable et régulière de bénéficier de la Sécurité sociale pour ses dépenses de santé. La mise en place de la CMU a été saluée unanimement comme un réel progrès en matière d'accès aux soins des plus précaires et comme une avancée significative des politiques de santé publique. Pourtant, un grand nombre d'associations ont déploré que la promesse d'universalité qui sous-tendait la CMU se trouve ainsi fractionnée entre "réguliers" et "irréguliers", soulignant ainsi les effets pervers d'une telle architecture ${ }^{4}$. Les mouvements associatifs engagés dans la défense des droits des étrangers ont très vivement contesté la distinction entre d'un côté, la couverture maladie universelle proprement dite et de l'autre, l'aide médicale d'État. Leur argumentaire s'est structuré autour du respect du principe de l'égalité de traitement et sur les textes internationaux ratifiés par la France, mais leur contestation est restée lettre morte. Même s'ils sont parvenus à certaines modifications de la loi, la remise en cause d'un partage entre l'accès à la CMU et à l'AME n'a pas reçu d'écho ${ }^{5}$. Aussi, sans relever spécifiquement du droit social des étrangers, la loi de juillet 1999 les concerne de premier chef : dans la mesure où ce dispositif est réservé aux individus résidant en France de façon stable et régulière, elle crée une catégorie à part, celle des étrangers en situation irrégulière.

6 Ce dispositif spécifique, l'AME, qui n'a pas d'équivalent dans les deux autres pays engagés dans la comparaison, permet à l'étranger en situation irrégulière de bénéficier d'une couverture maladie à condition de répondre à un certain nombre de critères : justifier de sa présence en France depuis au moins trois mois (depuis 2003), apporter la preuve de son identité et ne pas dépasser un certain plafond de ressource. Cependant, des écarts existent entre le principe et la pratique. 


\section{Une assistance médicale sous conditions}

7 D'abord, la complexité du fonctionnement des institutions et les pratiques parfois dissuasives des agents de la CPAM contribuent à retarder la jouissance de ce droit et par conséquent l'accès aux soins. Ensuite, l'AME constitue une prestation qui n'exige pas une contrepartie de cotisations. Relevant de l'assistance, elle est particulièrement soumise à des jugements moraux d'autant qu'elle s'applique à une catégorie d'étrangers dont le séjour est marqué par l'illégalité, ce qui les fait apparaître aux yeux de certains comme des délinquants ${ }^{6}$. À quoi s'ajoute un mode de financement qui rend visible une partie des coûts autrefois pris en charge par l'assurance maladie. Cela rend ce dispositif particulièrement sujet aux fluctuations gouvernementales ${ }^{7}$. Surtout, les bénéficiaires potentiels sont confrontés à la difficulté de réunir les pièces nécessaires à l'instruction de leur dossier, notamment en ce qui concerne les preuves relatives aux trois mois de présence sur le territoire français. Les propos d'une assistante sociale rencontrée dans une permanence d'accès aux soins de santé d'un hôpital parisien illustrent ces difficultés: "Les preuves de présence, c'est l'horreur! D'un département à l'autre, ça change. Un exemple: dans le 93, les cartes orange sont considérées comme des preuves de présence en France, alors que sur Paris non. Il faut trouver les preuves qui sont prises en compte par l'agent de Sécurité sociale, et c'est très aléatoire, c'est au cas par cas, ça dépend de l'agent de Sécurité sociale, de la CPAM, de la tête du client. C'est vrai que c'est plus complexe qu'à l'époque où il y avait une admission immédiate [...]." La question de la domiciliation semble également problématique pour des populations dont les possibilités et les conditions de logement demeurent aléatoires. Depuis le 20 juillet 20008, un décret signale que les associations doivent désormais révoquer la domiciliation de toute personne qui ne se serait pas présentée à leur structure depuis plus de trois mois. Cette disposition est révélatrice de ces tendances informelles et formelles à la restriction des droits; les réformes qui se sont succédées depuis la mise en place de l'AME sont allées dans le même sens. D'où l'importance de l'aide apportée par les assistants sociaux, tant pour faciliter l'obtention des droits des sans-papiers que pour permettre à l'institution hospitalière, plus particulièrement dans le cadre des permanences d'accès aux systèmes de soins (PASS), de les prendre en charge et de récupérer de manière rétroactive les frais engagés ${ }^{8}$.

\section{De la charge de la preuve au refus d'affiliation}

Longtemps effectif, l'accès aux soins des étrangers en situation irrégulière en GrandeBretagne a récemment été limité de manière drastique. En 2003, une campagne de presse a mis sur le devant de la scène la présence de migrants irréguliers et le poids qu'ils représentent pour le National Health Service (NHS), dans un climat de stigmatisation croissante des populations immigrées. Les médias ont dénoncé l'existence de supposés "health tourists", c'est-à-dire des migrants venus en GrandeBretagne pour se soigner aux frais du NHS, les accusant de contribuer à la faillite du système. Une consultation fut lancée à la suite de laquelle l'accès aux soins des irréguliers a été strictement limité par un ensemble de dispositions édictées en 2004 par le ministère de la Santé. Les soins de santé sont désormais restreints à l'urgence vitale et à une liste comprenant une dizaine de pathologies contagieuses (excluant le VIH). Au sein de l'institution hospitalière, les étrangers en situation irrégulière entrent dans la catégorie des visiteurs temporaires ou overseas visitors alors qu'ils bénéficiaient 
auparavant d'un accès de facto à l'ensemble des services de santé. Parallèlement, une nouvelle fonction a été mise en place, celle de l'overseas visitor manager, un agent administratif chargé d'établir en dernière instance si le patient a le droit d'être soigné ou non, soit gratuitement, soit en payant. Pour les soins de santé primaires prodigués par le general practitioner (GP), les nouveaux règlements de 2008 stipulent que le statut du résident doit être vérifié avant l'enregistrement du patient. Le GP garde un pouvoir discrétionnaire concernant l'enregistrement de nouveaux patients. Pour le patient en situation irrégulière il est difficile, en pratique, de prouver sa résidence, condition pourtant nécessaire dans la mesure où l'enregistrement chez le GP se fait en fonction de la résidence.

9 La spécificité du système suisse tient en partie à sa structure fédérale. En ce qui concerne la santé, la confédération légifère en matière d'assurance maladie ; au-delà de ce champ, ce sont les cantons qui définissent leurs propres modalités d'accès aux soins. Depuis 1996, la loi fédérale sur l'assurance maladie (LAMal) est entrée en vigueur avec pour objectif d'introduire un principe de solidarité dans la population : elle est devenue obligatoire pour toute personne domiciliée en Suisse. Théoriquement, les étrangers en situation irrégulière peuvent en bénéficier, mais un grand nombre d'assureurs ont interprété la loi de manière restrictive en refusant de les affilier. En vue de rappeler ces derniers à l'ordre, l'office fédéral des assurances sociales (Ofas) a stipulé dans une directive de 2002 que les assureurs doivent accepter toute demande d'adhésion provenant de personnes en situation irrégulière séjournant en Suisse depuis plus de trois mois. En réalité, cela n'a pas augmenté de manière significative le nombre d'assurés en situation irrégulière. En effet, la Croix-Rouge estimait en 2007 qu'environ 80 à $90 \%$ des sans-papiers"étaient dépourvus d'assurance maladie. Ce chiffre s'explique en partie par le fonctionnement même de l'assurance maladie. Les étrangers craignent de ne pas pouvoir honorer chaque mois le montant de l'assurance et d'être ainsi dénoncés aux autorités. Ils se trouvent dans cette situation paradoxale où l'accès à l'assurance maladie censée les protéger est, en lui-même, source de risque en raison des démarches que cela implique.

\section{Des volontés individuelles face à l'inertie des politiques publiques}

10 À l'exception du canton de Genève, ce sont les associations qui prennent en charge en grande partie l'accès aux soins des "sans-papiers" dépourvus d'assurance maladie. Dans certains hôpitaux, des dispositifs sont mis en place pour assurer leur prise en charge. Les démarches sont majoritairement impulsées et négociées par les professionnels en contact avec ce public étranger. Ces initiatives ne relèvent pas d'obligations institutionnelles mais de la bonne volonté d'individus. Les volontés individuelles sont primordiales dans la mise en place de ces dispositifs ce qui pose la question de leur pérennisation. Le système ne relève pas d'une organisation systématique, mais procède plutôt par des solutions pragmatiques et des arrangements entre les initiatives des personnels de santé et les autorités concernées. Pour chaque patient en situation irrégulière ne bénéficiant pas de l'assurance maladie des processus de négociation sont nécessaires pour permettre l'accès aux soins.

11 Dans les trois pays, le défaut d'affiliation des étrangers aux systèmes de soins s'accentue à mesure que les politiques d'immigration deviennent restrictives. Elles font 
apparaître au sein des structures médicales des acteurs chargés d'identifier les bénéficiaires et les non-bénéficiaires.

\section{Le même leitmotiv : la fraude, les abus, les économies}

12 En France, en Grande-Bretagne et en Suisse, ce sont les termes de fraude, d'abus et de restriction budgétaire qui servent à justifier la réduction des droits des personnes en situation irrégulière. En France, les réformes successives se sont appuyées sur ces arguments et, en décembre, deux députés de la majorité ont déposé une proposition de loi visant à réformer l'AME. L'exposé des motifs de la loi précise qu'il s'agit de lutter contre "les abus et les fraudes et de mieux contrôler ce gouffre financier". Dans cet objectif, il était prévu de centraliser les demandes d'AME au sein des mairies afin "de lutter contre les inscriptions multiples et les fraudes" et d'instaurer "un contrôle plus efficace de cette dépense de l'État". Le dernier rapport de l'IGAS sur l'aide médicale d'État avait pourtant démontré que la hausse de la dépense est avant tout due à celle du nombre de bénéficiaires et que la dépense moyenne par bénéficiaire restait contenue dans des limites assez proches de celle d'un assuré social'. En 2008, à nouveau, c'est le soupçon de fraude qui a justifié la mise en place de titres d'admission à l'AME non photocopiables. Plus récemment, un arrêté en date du 10 juillet 2009 met en place une carte plastifiée pour assurer une "meilleure sécurisation".

13 En Suisse, la thématique de l'abus des étrangers a constitué l'argument principal pour restreindre les droits sociaux des demandeurs d'asile. La révision partielle de la loi fédérale sur l'asile (LAsi) qui est entrée en vigueur en 2008 exclut de l'aide sociale les personnes dont la décision de renvoi est exécutoire et auxquelles un délai de départ a été imparti. L'exclusion de l'aide sociale concerne déjà, depuis avril 2004, les personnes faisant l'objet d'une décision de non-entrée en matière (NEM) ${ }^{10}$. Ces nouvelles dispositions de la loi ont invoqué - de manière fallacieuse eu égard à la réalité l'existence d'“abus" afin de justifier les mesures restrictives introduites dans la loi ou dans la pratique à l'encontre des étrangers ${ }^{11}$.

14 En Grande-Bretagne, le discours sur la crise de financement de la NHS et sur l'illégitimité des migrants irréguliers à y prétendre semble bien installé dans les médias. Dans le débat public sur l'immigration, on note une confusion entre les immigrés et les demandeurs d'asile, ces derniers étant accusés de plus en plus fréquemment d'être des "migrants économiques" et de "faux demandeurs d'asile" venus profiter du système social britannique. Nombre d'entretiens ont aussi souligné le rôle clé que joue le souvenir encore vivace des attentats de 2005 dans ce rejet de l'étranger.

\section{La peur de l'appel d'air}

15 Derrière l'évocation de la fraude se profile l'idée que les systèmes de santé des pays occidentaux exerceraient une forte attraction sur le reste du monde, créant ainsi un "effet d'appel d'air" sur les migrations. La dernière enquête menée par l'observatoire européen sur l'accès aux soins des étrangers en situation irrégulière en Europe montre une réalité tout autre. Parmi les personnes interrogées dans les centres de soins de Médecins du Monde de onze pays d'Europe, $6 \%$ d'entre elles avancent la santé comme raison de leur migration, tandis que $56 \%$ affirment avoir quitté leur pays pour des 
raisons économiques et que $26 \%$ expliquent leur départ par des motifs politiques, religieux ou ethniques. En France, où existe pourtant un dispositif permettant l'accès des sans-papiers aux systèmes de soins, seules $10 \%$ des personnes en situation irrégulière avancent la raison de la santé pour la migration, un chiffre assez proche du pourcentage que l'on retrouve en Grèce $(8,5 \%)$, pays qui est l'un des plus défavorables à l'accès aux soins de ces populations. Les auteurs du rapport notent ainsi que les raisons de départ avancées n'ont pas de rapport direct avec la facilité présumée des systèmes de soins et ils concluent : "On n'observe donc pas dans cette enquête l'effet d'appel d'air de la migration tant mis en avant dans certains discours politiques ${ }^{12}$."

En France, plusieurs recherches menées à partir d'interrogations différentes parviennent à des conclusions semblables. L'étude conduite dans le Val-d'Oise sur les demandes de séjour pour raison médicale entre 2002 et 2003 montre que la progression des demandes constatées à cette période de l'enquête est davantage le reflet des flux migratoires que de la volonté des étrangers de se faire soigner en France ${ }^{13}$. Fondée sur les données de l'Enquête santé de 2002-2003, la recherche sur les inégalités dans l'accès à la santé touchant les populations immigrées suggère l'existence d'un effet de sélection à la migration : parmi ceux qui migrent, on compte d'abord des personnes en bon état de santé ou issues de milieux privilégiés. Dans un autre registre, le rapport d'activité du Comede de 2008 souligne qu'un faible pourcentage (6\%) de migrants était au courant de leur pathologie avant de décider de leur départ.

17 Ces chiffres de même que les enquêtes menées vont à l'encontre des discours récurrents sur l'attraction des systèmes de soins comme principal moteur de l'immigration, discours qui relèvent plus du fantasme que de la réalité. Reste qu'en France, en Grande-Bretagne et en Suisse, ce sont ces arguments qui continuent de justifier les mesures et les projets de réduction des droits des étrangers en situation irrégulière. Au-delà des raisons budgétaires, c'est la légitimité même de ces droits qui est mise en cause: "Lorsque la fraude invoquée à l'encontre de quelques-uns justifie la réduction de l'aide pour tous, la légitimité de l'aide est autant visée que la réduction des coûts ${ }^{14}$." Dans les trois pays, se déploie la même rhétorique du welfare chauvinism qui fait de l'étranger en situation irrégulière un concurrent déloyal du citoyen européen pour l'accès au marché du travail ou pour la répartition des biens sociaux ${ }^{15}$.

\section{Politiques d'immigration et dilemmes du personnel soignant}

Les personnels soignants en contact direct avec des étrangers en situation irrégulière ont mis l'accent sur les effets des restrictions en matière de politiques d'immigration sur l'accomplissement de leur mission. Bon nombre de professionnels ont ainsi le sentiment que leur pouvoir d'action au sein de l'institution hospitalière se réduit. Leur marge de manœuvre s'est affaiblie, elle se heurte aux impératifs financiers de l'institution auxquels s'ajoutent les contraintes qu'imposent les dernières politiques d'immigration. Elles viennent directement interférer dans les parcours de soins de leurs patients. À Lausanne, on a rencontré un médecin encore sous le choc de l'expulsion d'un de ses patients : "On l'a pris en charge alors qu'il était dans une situation de grande précarité, séropositif avec une tuberculose disséminée et sans-papiers. On a commencé un traitement et on vient de m'apprendre qu'il a été expulsé. On ne peut pas faire cela, même s'il $y$ a des lois, des politiques. On arrive aux limites de ce qi est admissible." 

$\mathrm{du}$ soin, soient inféodées à un mode de décision guidé par des impératifs d'ordre administratif, financier et politique. Un médecin d'un hôpital parisien évoque les "choix terrifiants" auxquels il se trouve confronté lorsqu'il est face à des patients qui n'entrent pas dans le droit commun : "Nous, ce qu'on nous demande quand on a des patients qui n'ont pas l'AME c'est de choisir entre ce qui est urgent ou pas urgent". De même en Grande-Bretagne, les soignants ont le sentiment que les administratifs remettent en question leur autonomie en imposant comme condition d'accès aux soins le statut juridique légal des patients: "On découvre que les médecins n'ont pas la liberté de faire ce qu'ils veulent, le pouvoir administratif dans les hôpitaux est plus fort..." (Travailleur social, ONG médicale, Grande-Bretagne, Londres).

ces multiples situations, des réponses sont apportées localement selon la mobilisation et la bonne volonté de l'équipe de soignants et de travailleurs sociaux. En France, à l'hôpital Saint-Louis (Paris), par exemple, pour le cas des étrangers qui ne peuvent pas bénéficier de l'AME, les personnels de la permanence d'accès aux soins "Verlaine" ont mis en place un groupe de réflexion "Éthique et précarité" sur la prise de décision pour l'entrée dans les PASS des étrangers ne relevant pas d'un accès immédiat à une couverture sociale. Il s'agit de définir un code de conduite qui préserve la qualité et l'éthique de leur travail car "les décisions peuvent aller d'un extrême à l'autre, du retour au pays sans examiner en détail la situation, à l'intégration immédiate et systématique dans le dispositif de soins" et "la décision varie ainsi considérablement d'une personne à l'autre que ce soit chez le médecin ou chez les travailleurs sociaux ${ }^{16 " . ~}$

\section{Déterminer l'urgence vitale : le soin face à la tutelle administrative}

21 À Lausanne, en 2000, afin de réagir devant les dernières dispositions de la loi, un groupe de médecins de la policlinique médicale universitaire (PMU) et du centre hospitalier universitaire vaudois (CHUV), épaulé par un juriste, un éthicien et des gestionnaires de ces institutions, a mis en place une Commission chargée de définir par des critères médicaux quels sont les plus vulnérables parmi les précaires qui doivent bénéficier d'une aide. Les personnels de santé se trouvent donc confrontés à des décisions difficiles. Patrick Bodenma, président de cette Commission, souligne ainsi : “ Le médecin n'est pas au-dessus des lois, cependant la dureté de certaines lois et le fait qu'elles se rapprochent d'une transgression des droits fondamentaux entraînent chez le soignant un véritable cas de conscience. [...] Ce sera aux limites de sa conscience que le médecin essaiera de déterminer qui, parmi les vulnérables, est encore plus vulnérable." Plus récemment, en 2005, le CHUV et la PMU ont mis sur pied la "Commission des populations sociales" composée de 19 personnes de différents corps de métiers représentés au sein de l'institution (médecins, juristes, administrateurs services sociaux, éthiciens, service financier). Pour pallier l'absence d'un modèle de référence en Suisse, l'institution a décidé de se positionner en "centre d'expertise" pour formaliser des procédures dans les situations où l'état de santé d'étrangers en situation irrégulière nécessiterait une hospitalisation. ${ }^{17}$ La démarche est la suivante : lorsque l'hôpital se trouve en présence d'un étranger en situation irrégulière, sans assurance maladie et gravement malade, son cas est signalé à la Commission par courrier électronique. Elle dispose alors de 48 heures pour décider si le patient sera pris en charge ou non.

Hommes \& migrations, 1284 | 2010 

l'on concède, et non pas de l'application d'une règle de droit impartiale. Ils sont parfois conduits à s'investir d'une manière individuelle qui déborde leurs obligations professionnelles et restent dubitatifs face à ces accommodements qui procèdent d'arrangements susceptibles d'être toujours remis en question. Un médecin d'un hôpital parisien expliquait que, pour mener à bien sa mission, elle devait accomplir des démarches qui ne relevaient pas initialement de ses prérogatives : "Il y a des tas de dossiers perdus à la préfecture. On avait une patiente VIH très mal en point et à la préfecture on nous a dit que le dossier était perdu. On a refait le dossier avec l'assistante sociale, on l'a envoyé, on a appelé, on a rappelé et on leur a dit qu'on avait reçu l'accusé de réception.... Finalement, ils nous ont dit qu'ils l'avaient reçu." soignants ou des administratifs en faveur de tel ou tel patient apparaît comme décisive. "Actuellement pour orienter un patient irrégulier, on peut toujours travailler en réseau, trouver un intermédiaire, et les hôpitaux nous écoutent. Mais je sens que l'étau se resserre. Pour l'instant, on peut toujours discuter, utiliser nos contacts. Mais avec le temps, qui sait si ce sera encore possible à l'avenir? Il ne faut pas se contenter de ce type de fonctionnement ad hoc." (Travailleur social, Grande-Bretagne, ONG de défense des migrants africains séropositifs, Londres).

\section{Sortir de son rôle pour mener à bien sa mission}

Ainsi, les professionnels de santé mobilisent d'autres ressorts d'action que la logique purement professionnelle afin que leurs patients en situation irrégulière puissent avoir un accès effectif au système de soins. La connaissance des politiques d'immigration s'avère à cet égard déterminante. Elle constitue la base même des conseils qu'ils jugent utiles de prodiguer, même lorsqu'ils considèrent qu'en agissant ainsi, ils sortent de leur rôle médical. C'est le cas de cette médecin qui regrette d'être intrusive dans la vie de ses patients quand elle essaie d'expliquer à certains d'entre eux que leur dernière chance de rester en France et de se faire soigner est d'accepter de renoncer à se voir reconnaître légitimement comme réfugié. Elle leur conseille alors de mettre en avant leur maladie plutôt que les menaces qui les ont poussés à l'exil. Cet exemple recoupe les analyses de Didier Fassin à propos du régime de bio-légtimité traduisant le fait que la maladie est devenue plus légitime que l'asile politique pour celui qui prétend à un titre de séjour ${ }^{18}$. "Au lieu de faire une demande de réfugié politique qui va être refusée, je conseille fortement de faire une demande de régularisation pour soins. Je pense à un patient qui est menacé dans son pays et qui a l'hépatite B. Je crois qu'il aurait plus de chance d'être régularisé pour soins que d'obtenir un statut de réfugié politique, alors j'insiste. Mais ce n'est pas facile, je m'engage. Et puis, si jamais ça ne marche pas, il peut m'en tenir responsable. Même si je sais qu'il n'a presque aucune chance d'être réfugié, eh bien, il faut quand même que je me débrouille avec ma conscience!"

Il arrive à des médecins d'avoir, malgré eux, à prendre des décisions délicates et d'autant plus complexes qu'elles intègrent des considérations ayant directement trait aux politiques d'immigration. C'est plus particulièrement le cas en France pour les demandes de régularisation pour soins qui révèlent l'implication étroite entre politique d'immigration et questions de santé. Le témoignage de ce médecin montre comment l'une vient perturber l'autre: "On me demande de faire un certificat où je dois dire la vérité. 
Et je dis la vérité! Je décris la pathologie et j'écris la formule qui dit qu"elle nécessite une prise en charge dans un établissement hospitalier public et une surveillance régulière et que le retour dans son pays le mettrait dans une situation de danger'. Mais si le type se présente avec ce certificat, l'inspecteur de santé de la préfecture va dire 'Vous êtes malade mais vous pouvez vous faire soigner à Bamako'. Et moi, je sais qu'il ne veut pas retourner au Mali. Or maintenant, à la préfecture, ils savent qui il est, où il habite... Ce sont toutes ces considérations que j'ai chaque fois en tête."

En France, en Grande-Bretagne et en Suisse, l'engagement personnel des professionnels de santé, au-delà même de leurs prérogatives, s'avère crucial pour permettre aux étrangers en situation irrégulière d'accéder aux soins. Néanmoins la dimension structurelle des systèmes et des dispositifs de santé dans l'un ou l'autre de ces pays favorise des postures sensiblement différentes. De manière schématique, on peut en distinguer trois principales, non exclusives les unes des autres. En France, l'existence d'un dispositif tel que l'AME laisse ouverte la possibilité, pour les professionnels de santé, d'instaurer une relation avant tout axée sur le soin et d'endosser en cela une posture médicale. Dès lors que les étrangers en situation irrégulière bénéficient d'une protection médicale (l'AME), ils peuvent - à tout le moins en principe - être traités comme des patients ordinaires sans que leur précarité sociale et juridique ne soit placée au premier plan. En Suisse (Lausanne, canton de Vaud), le système d'accès aux soins des étrangers en situation irrégulière favorise une posture éthique au sens où le personnel soignant choisit d'exercer gratuitement dans des associations ou de développer des dispositifs, ce qui relève davantage du don, de la sollicitude, de la compassion. Dans ce cas, ce n'est pas le droit mais l'éthique qui définit la relation soignant patient. En Grande-Bretagne, face à la restriction drastique des droits des étrangers en situation irrégulière, les soignants sont amenés à décider si oui ou non ils souhaitent s'engager pour leur assurer des soins effectifs. Ces actes individuels les contraignent à transcender une posture strictement médicale, y compris lorsque cette dernière est motivée par des considérations éthiques, pour emprunter une posture politique. Il s'agit pour eux de prendre la responsabilitéde poser délibérément un acte militant qui, s'il n'est pas interdit, va néanmoins à contre-courant de la législation en vigueur et plus largement de l'opinion publique.

Rares sont ceux qui viendraient contester que la santé soit un des biens humains qu'il faille protéger universellement. Comme le souligne Didier Fassin, "la maladie et la souffrance offrent une base minimale pour un consensus politique de tolérance et même de reconnaissance de l'étranger ${ }^{19}$ ". Cependant, ce consensus est de plus en plus mis à mal en pratique lorsqu'il faut trancher entre des situations d'urgence vitale et d'autres qui ne mettent pas en péril la vie de manière immédiate. C'est pourtant ce à quoi les politiques d'immigration poussent, toujours davantage, les professionnels de santé.

\section{NOTES}

1. Lochack Danièle, Les Droits de l'Homme, Paris, La Découverte, $3^{\mathrm{e}}$ ed., 2009. 
2. Guiraudon Virginie, "Une obsession partagée", Plein droit, "Une Europe de rejet", n57, juin 2003.

3. Cet article est tiré d'une enquête financée par la Drees-MiRe (appel d'offres "MigrationProtection sociale", 2007) menée sous la direction de Serge Paugam (Centre Maurice Halbwachs, Équipe de recherche sur les inégalités sociales [ERIS] CMH, UMR 8097 CNRS, EHESS, ENS). Les participants sont?: Sandro Cattacin (Université de Genève), Milena Chimienti (Université de Genève), Myriam Hachimi Alaoui, (Université du Havre/Cirtai), Alexandra Nacu, (Centre de sociologie des organisations). Les données de cet article reposent sur une enquête qualitative qui s'est déroulée dans des centres de soins humanitaires ou dans des structures institutionnelles dans lesquelles des dispositifs pour l'accueil des populations précaires sont mis en place. Par conséquent, et il est important de le souligner, les personnes avec lesquelles nous avons mené des entretiens manifestent d'emblée un intérêt pour le sort des populations précaires.

4. Veïsse Arnaud, "Les salles d'attente de l'universel", Vacarme, n 17, 2001.

5. Voir à propos de la CMU, l'article de Didier Maille et de Adeline Toullier, "Les dix ans de la CMU. Un bilan contrasté pour l'accès aux soins des migrants", Hommes et Migrations, "Santé et droit des étrangers", n 1282, nov-déc 2009, p. 24-33.

6. Nous renvoyons à ce sujet à l'ouvrage dirigé par Serge Paugam, Repenser la solidarité, Paris, PUF, 2007.

7. Voir à ce propos: Gotman Anne, Assier-Andrieu Louis, "Immigration et accès aux droits sociaux : enquête sur les logiques discriminatoires", Fonds d'action et de soutien pour l'intégration et la lutte contre les discriminations-CEPEL, 2004.

8. Parizot Isabelle, Soigner les exclus, Paris, Puf, "Le lien social”, 2003.

9. Gissler E., Azoulay J., Chambaud L., Corlay D., Havard H., Sourlas P., Rapport sur la gestion de l'aide médicale d'État, Paris, IGF-IGAS, 2007.

10. Une décision de non-entrée en matière signifie que, pour des raisons formelles, il ne sera pas donné suite à la demande d'asile. La demande déposée ne fait l'objet que d'un examen limité. Les personnes concernées doivent en règle générale quitter immédiatement la Suisse.

11. Tafelmacher Christophe, "La 'chasse aux abus', une arme pour démanteler les droits", in Schmidlin et al. (dir.), La Politique d'asile à la dérive, Lausanne, Éditions d'en bas, 2004.

12. Chauvin Pierre, Parizot Isabelle, Simonnot Nathalie, L'accès aux soins des personnes sans autorisation de séjour dans 11 pays d'Europe, Observatoire européen de l'accès aux soins de Médecins du Monde, 2009.

13. La Ruche G., Brunet E., "Demandes de séjour pour raison médicale des malades étrangers dans le département du Val-d'Oise de 1999 à 2003", Société française de santé publique, vol. 1, n 18, 2006, p. 119-130.

14. Gotman Anne, Assier-Andrieu Louis, op. cit.

15. Denis Duez, L'Union européenne et l'immigration clandestine, Université de Bruxelles (eds.), 2008, p. 180.

16. George Claire, Consultation sans rendez-vous/PASS. Soins primaires/précarité dans le nord-est parisien, Hôpital Saint-Louis, 5 décembre 2007, document interne.

17. Rapport d'activité de la Commission des populations vulnérables, Lausanne, document interne, 2008.

18. Fassin Didier (avec D. Memmi), Le Gouvernement des corps, Paris, Éditions de l'École des hautes études en sciences sociales, Paris, 2004.

19. Fassin Didier, "Santé et migration. Les vérités politiques du corps", Cahiers de l'URMIS, n 5, 1999. 


\section{AUTEURS}

MYRIAM HACHIMI ALAOUI

Maître de conférences, Université du Havre/Cirtai, chercheur associée à l'équipe ERIS du Centre Maurice Halbwachs.

\section{ALEXANDRA NACU}

Chercheur associée au Centre de sociologie des organisations (Cnrs-Sciences Po). 